\title{
Ultrasound evaluation of infants suspected to have biliary atresia with emphasis on the triangular cord sign: The Lagos experience
}

\author{
Osuoji R.I ${ }^{1}$, Akinola R.A ${ }^{2}$, Sebanjo I.O ${ }^{3}$, Ajayi O.I ${ }^{4}$ \\ ${ }^{1}$ (Department of Surgery, Paediatric Surgery Unit/College of Medicine, Lagos State University Teaching \\ Hospital, Ikeja, Lagos, Nigeria) \\ ${ }^{2}$ (Department of Radiology/ College of Medicine, Lagos State University Teaching Hospital, Ikeja, Lagos, \\ Nigeria) \\ ${ }^{3}$ (Department of Paediatrics and Child Health/ College of Medicine, Lagos State University Teaching Hospital, \\ Ikeja, Lagos, Nigeria) \\ ${ }^{4}$ (Department of Radiology/ College of Medicine, Lagos State University Teaching Hospital, Ikeja, Lagos,
} Nigeria)

\begin{abstract}
The morbidity and mortality from biliary atresia cannot be overemphasized. Therefore, its early detection especially using a non invasive means will go a long way in preventing liver cirrhosis, liver failure and even death from this disease entity. This study was done to highlight the abdominal scan findings in infants suspected to have biliary atresia, demonstrate the Triangular Cord sign (TC) and to assess how many of these were confirmed at surgery. It is a prospective study of 25 consecutive jaundiced infants sent to the Radiology department of a Teaching Hospital for abdominal ultrasound scan, on account of persistent elevated conjugated bilirubin and thus the suspicion of biliary atresia. The Triangular cord sign, liver size and echotexture, gallbadder longitudinal diameter, pre and postprandial were assessed. Infants that were highly suspected for biliary atresia were operated and findings were correlated with ultrasound findings. About a third of the infants scanned had a positive Triangular cord sign, only three of whom were found to be positive at surgery. The Triangular cord width ranged from $0.07-4.7 \mathrm{~mm}$, with a mean of $2.62 \pm 1.23 \mathrm{~mm}$. This study confirmed that TC measuring 3.5 - 4mm or more on ultrasound is diagnostic of biliary atresia.
\end{abstract}

Key Words: Biliary Atresia, Hepatitis, Congugated Hyperbilirubinaemia, Triangular Cord Sign.

\section{Introduction}

Studies have shown that extrahepatic biliary tract obstruction, idiopathic hepatitis, and bacterial infections were common causes of conjugated hyperbilirubinaemia in Nigerian infants [1]. Neonatal cholestasis is still a major diagnostic challenge despite increasing knowledge about its pathogenesis and one of its most common cause is biliary atresia.

Biliary atresia is a congenital biliary disorder, commonly causing progressive and obstructive pathological changes in the intra and extrahepatic ducts, secondary to inflammation, and often causing progressive fibrosis and liver damage, resulting in cirrhosis of the liver and consequent liver failure $[\mathbf{2 , 3 , 4 , 5 ]}$ It is characterized by absence or severe deficiency of the extra-hepatic biliary tree [6], and accounts for over half of children who undergo liver transplantation [5]. It is thought to affect 1 in 10,000 - 15,000 newborn infants, with a recognized male preponderance $[\mathbf{3 , 5}$. These infants may be normal at birth. However symptoms develop at 2 weeks to 2 months of life, presenting with jaundice, dark yellow or brown urine, clay colored stools and hepatomegaly [5].

Prompt and accurate diagnosis is essential for early operative intervention, efficient treatment and a good prognosis to prevent liver cirrhosis in such neonates $[\mathbf{5 , 6}]$. To aid the diagnosis of biliary atresia, an abdominal ultrasound and hepatobiliary iminodiacetic acid (HIDA) scan may be used [6].

Most of our patients present late in Nigeria when they might have developed cirrhosis [1]. Several investigators have described the Triangular Cord sign (TC) as a useful and specific ultrasound (USS) finding for the differential diagnosis of biliary atresia from neonatal hepatitis [7]. There are some evidences that TC sign, i.e triangular structure located cranial to the portal vein bifurcation on ultrasonographic examination, is suggestive of biliary atresia (BA) in suspected cases [6]. Imanieh et al [6] concluded in their study, that TC sign is more accurate than hepatobiliary scan and has acceptable sensitivity and specificity for diagnosis of BA.

The diagnosis of BA in neonates in this resource limited part of the world has been quite difficult. This study was therefore done to summarize the ultrasonographic findings in neonates with cholestatic jaundice suspected to have biliary atresia, to demonstrate the obliterated fibrous ductal remnant in the porta hepatis (TC sign) and attempt to find out how many of the positive ultrasound diagnosed TC sign was proven at surgery. There is a dearth of such a study in Nigeria as revealed by our search through literature. This could help 
augment early and accurate diagnosis of biliary atresia in this environment in future and thus reduce the morbidity and mortality from this disease entity.

\section{Materials And Methods}

2.1 Methodology: A prospective, descriptive study of 25 consecutive persistently jaundiced infants, with conjugated hyperbilirubinemia, suspected to have BA, sent to the Radiology department of the Lagos State University Teaching Hospital for an abdominal ultrasound scan. This was done as part of their management work up, with a 10.0-MHz transducer of a Logiq 5 Expert. GE ${ }^{\circledR}$.

The study was from June 2011 to November 2012. All the parents voluntarily signed consent forms, agreeing with the procedure and the hospital research and ethics committee approved the conduct of the study. The Triangular cord sign, liver size and echotexture, pre and postprandial gallbladder longitudinal diameter were assessed and documented. Those that were highly suspected for BA, (when the TC measured $3.5 \mathrm{~mm}$ with no gallbladder seen or more than $3.5 \mathrm{~mm}$ with or without a gallbladder seen), were operated on consent and the operative findings were correlated with the ultrasound findings.

2.2 Operational definition: The TC was defined as a triangular, or tubular, echogenic area seen immediately anterior to the portal vein bifurcation in the region of the porta hepatis.

We used Hee-Jung Lee et al's [7] criteria. The presence of triangular cord, the size and echo of liver, the presence or absence of the gallbladder, the changes in gallbladder size, pre and postprandial were observed and recorded as done by Shi-Xing Li et al [2].

\subsection{Technique}

All USS examinations were performed by the radiologist and the registrar. A real-time ultrasound machine, Logiq 5 Expert. , with a high frequency 10-MHz linear transducers was used. The infants were fasted for 4 hours before the general abdominal scan. The liver, spleen, kidneys and the porta hepatis were also routinely scrutinized.

The thickness of the echogenic area anterior to the right portal vein (EARPV) was measured using the Hee-Jung Lee et al [7], method. The sole criterion for the TC sign in this study was a thickness of $3.5 \mathrm{~mm}$ or more on a longitudinal or transverse scan, Fig 1a and b.
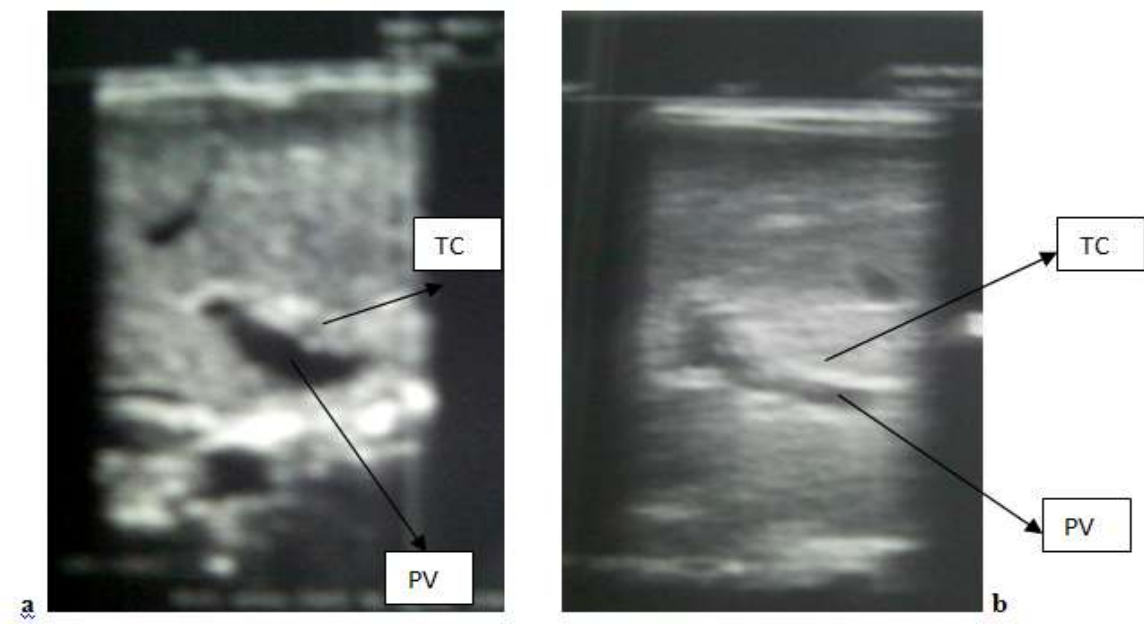

Fig 1 Abdominal scan in the right hypochondrium showing a tubular (a) and triangular (b) echogenic area anterior to the bifurcation of portal vein, at the porta hepatis - TC Sign. PV is the portal vein.

Measurements were obtained using electronic calipers and a thickness of $3.5 \mathrm{~mm}$ was chosen as the upper limit of normal, taking into consideration all normal possible structures that could be seen anterior to the right portal vein, such as the anterior wall of the right portal vein, anterior wall of the right hepatic artery and the common hepatic duct.

For consistency, measurements were obtained in the proximal part of the portal vein just before its bifurcation, at the widest part of the echogenic structure and perpendicular to the plane of the scan, even when the TC was irregular. At least three measurements were taken for each patient and the average calculated and documented.

The diagnosis of BA was made when the TC sign was present on USS with or without visualization of the gallbladder. Also, TC measurements of $3.5 \mathrm{~mm}$ were considered as suggestive of BA, when the subject's gallbladder was not demonstrated. Patients with BA whose parents consented to operation had excision of the fibrous cord and portoenterostomy - Kasai operation and a liver biopsy was also taken to complete the procedure. The USS findings were compared with surgical findings. 


\subsection{Data Analysis}

All data was entered into a Microsoft word excel spread sheet and analyzed using the statistical package for social sciences, version 16 for Windows Microsoft, Chicago, Illinois. Descriptive statistics (minimum, maximum, mean, standard deviation) were calculated for continuous variables. Percentages and proportions were determined for categorical variables. Charts were used for graphical illustrations.

\section{Results}

The age range of the twenty five study subjects was 3 to 36 weeks with a mean of the $11.27 \pm 9.13$ weeks. More than half of them, 52\%, were 3-8weeks old, while $12 \%$ of them were more than 20 weeks, Fig 2.

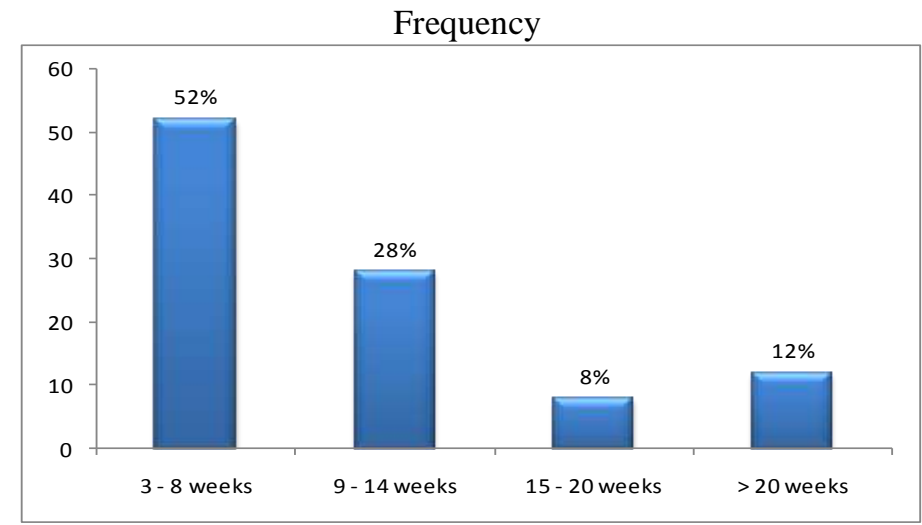

Age in weeks

Fig 2 Age distributions of study subjects

Majority of them, 60\% were females, Fig 3 with all of them presenting with jaundice, conjugated hyperbilirubinaemia, pale stools, and one each presenting with dark urine, abdominal distension, and hepatomegaly.

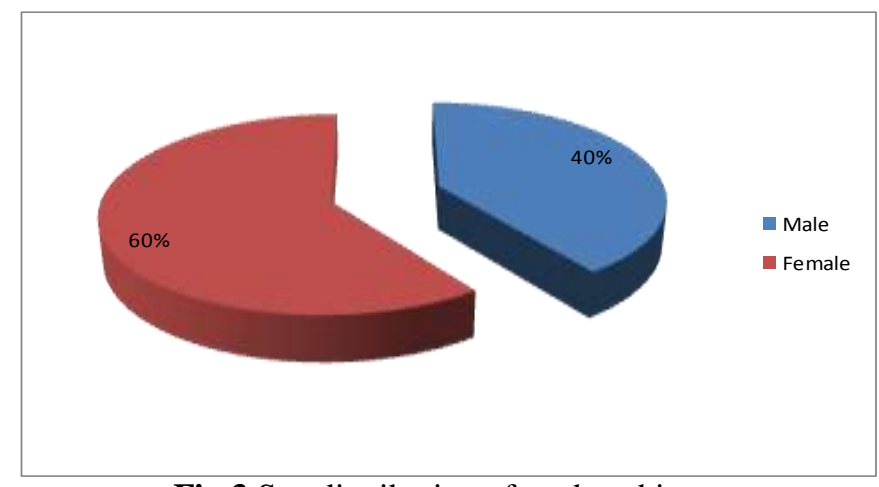

Fig 3 Sex distribution of study subjects

The onset of jaundice was below 1 week of age in $16 \%$ of the cases and above 10 weeks in $64 \%$. The other mothers, $20 \%$ could not remember exactly when jaundice commenced.

The average size of liver observed among the neonates was $7.54 \pm 1.60 \mathrm{~cm}$, with the largest measuring $11.8 \mathrm{~cm}$. Seventy two percent of the patients had liver larger than $6.5 \mathrm{~cm}$ in span. They were homogenous in $22(88 \%)$ of the infants, and heterogenous in $3(12 \%)$ of them. Liver texture was harsh in over half of them, $52 \%$, Fig $4 \mathbf{a}$ and b.
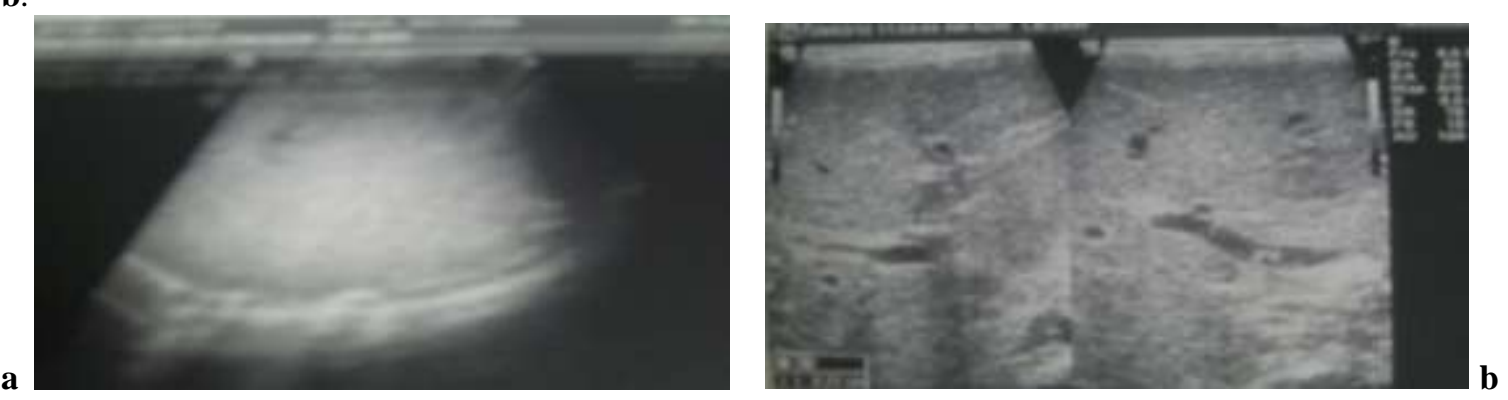
Fig. 4a and b A longitudinal scan of the liver showing a homogenously (a) and heterogeneously (b) harsh echopattern liver

The gallbladder was normal in about half, $52 \%$ of the infants and there was change in the sizes pre and postprandial. One of them had a stone casting a strong acoustic shadow within it, Fig 5. The gallbladder was small in 2 infants and was absent in 9 of them. One of the gallbladders did not shrink at all postprandial.

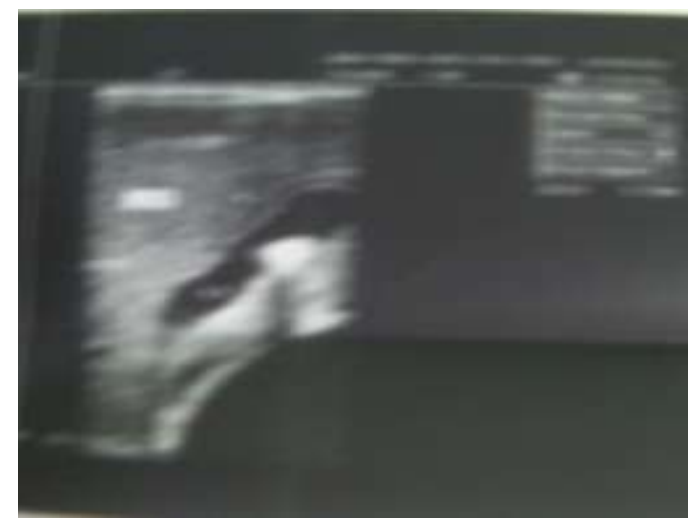

Fig 5 Showing a gallbladder with a stone within it casting a strong acoustic shadow

TC measurement was positive, i.e $\geq 3.5 \mathrm{~mm}$ in $36 \%$ cases and most, $64 \%$ were negative as revealed by ultrasound, Fig 6. The minimum width of TC was $0.07 \mathrm{~mm}$, while the maximum was $4.7 \mathrm{~mm}$, with a mean width of was $2.62+1.23 \mathrm{~mm}$.

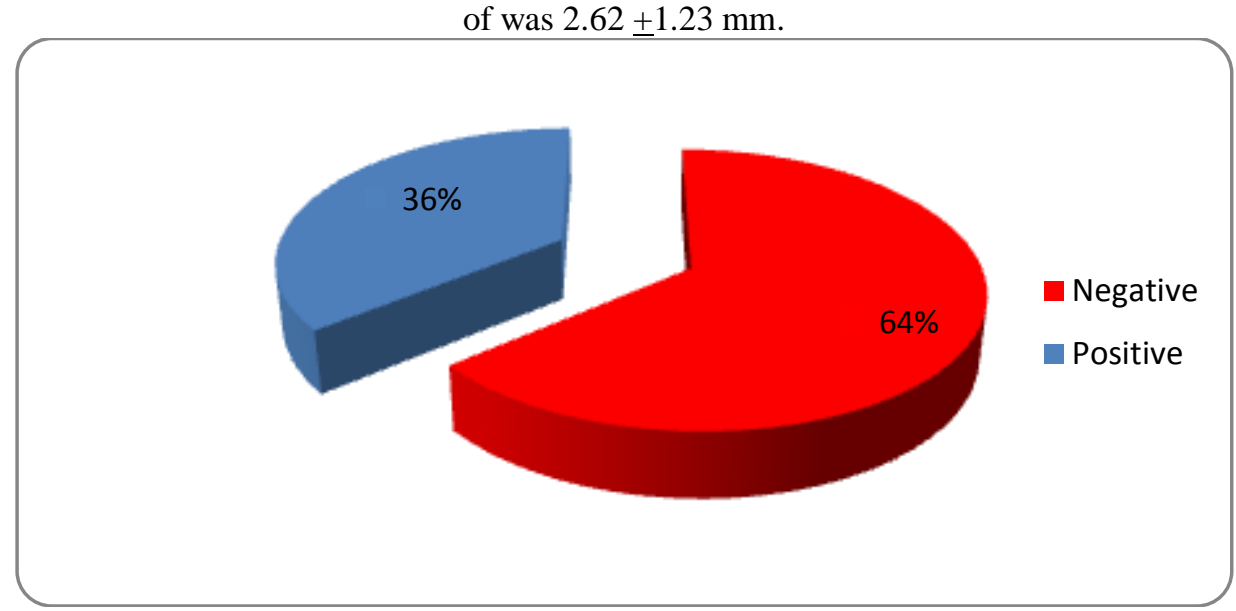

Fig 6 Pie chart demonstrating the distribution of the TC width

The Common Bile Duct (CBD) was not seen in $28 \%$ of the neonates and among the $72 \%$ in whom the CBD was seen, its mean size was $0.46+0.42 \mathrm{~cm}$.

Inferior Vena Cava and Portal vein were seen in all the infants.

Spleen was normal in all except one case, in whom it was enlarged.

Majority, $72 \%$ of the kidneys were normal, echogenic in $20 \%$ neonates, Fig 7; echogenic with prominent renal pyramids in $4 \%$ and atrophic right kidney in $4 \%$ of cases.

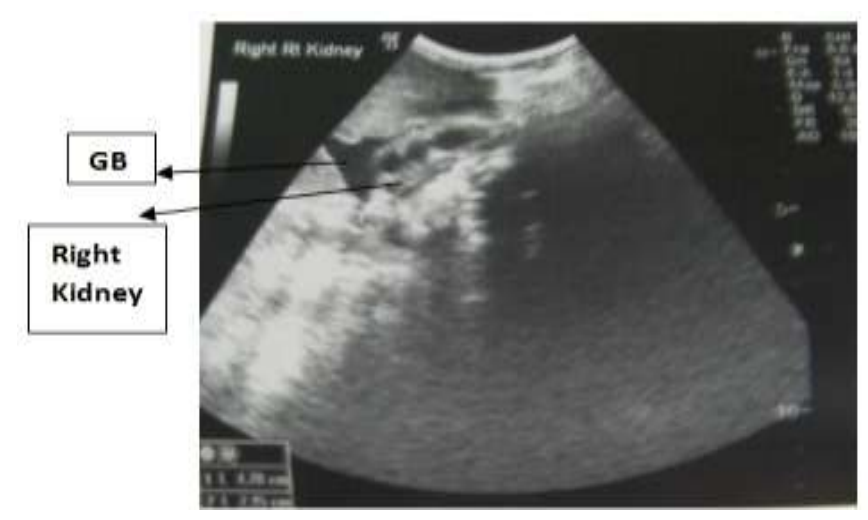


Fig 7 Showing an echogenic right kidney with the gallbladder (GB) adjacent to it. Ascites was not seen in any of the infants.

An ultrasonic diagnosis of hepatitis was made in $12(48 \%)$ of the neonates, biliary atresia in $9(36 \%)$ of them, cirhosis; 1 (4\%), while $3(12 \%)$ of the infants were classified as normal. Clinical improvement of neonatal hepatitis was verified by the patient's recovery from jaundice and the normalization of laboratory values during clinical follow-up period.

The other incidental findings revealed were thick irregular inner wall of gall bladder, 3.8\%; dilated branching biliary tree at porta hepatis, 3.8\% Fig 8; dilated intrahepatic ducts, 3-8\%;

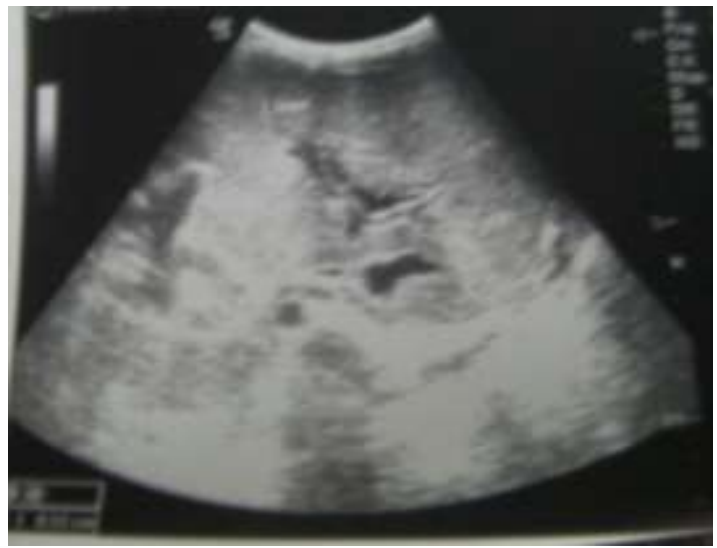

Fig 8 Showing dilated intrahepatic ducts

choledochal cyst, $3.8 \%$; gall stone, $3.8 \%$; harsh kidney echoes, $11.5 \%$; echogenic prominent renal pyramids, $3.8 \%$; large cystic structure in right flank, suggesting a pelviureteric junction obstruction (PUJ), 3.8\%; prominent portal vein, $3.8 \%$; cystic structure around the right kidney which looked like another gall bladder and thus a suggestion of a duplicated gall bladder.

Table 1 Others Ultrasound Findings

\begin{tabular}{|lcc|}
\hline Findings & Frequency & Percent \\
\hline Harsh echogenic Kidneys & 3 & 11.5 \\
$\begin{array}{l}\text { Echogenic prominent renal } \\
\text { pyramids }\end{array}$ & 1 & 3.8 \\
$\begin{array}{l}\text { Large cystic structure in right } \\
\text { flank to rule out PUJ } \\
\text { obstruction }\end{array}$ & 1 & 3.8 \\
$\begin{array}{l}\text { Dilated branching biliary tree } \\
\text { at porta hepatis } \\
\text { Dilated intrahepatic ducts }\end{array}$ & 1 & 3.8 \\
$\begin{array}{l}\text { Choledochal cyst } \\
\text { Irregular inner wall of gall }\end{array}$ & 1 & 3.8 \\
bladder, with a stone & 1 & 3.8 \\
$\begin{array}{l}\text { Cystic structure around the } \\
\text { right kidney which looked like } \\
\text { another gall bladder, duplicated } \\
\text { gall bladder }\end{array}$ & 1 & 3.8 \\
\begin{tabular}{l} 
Prominent portal vein \\
\hline
\end{tabular} & 1 & 3.8 \\
\hline
\end{tabular}

Fifteen of the neonates were treated conservatively, though about 5 of them were lost to follow up. Three, $12 \%$ were confirmed to have biliary atresia at surgery (Kasai's operation), one of these three proven cases was also confirmed at histology to have cirrhosis. The thick fibrous cord anterior to the bifurcation of the portal vein were confirmed and removed. The TCs that were confirmed positive at surgery measured, $3.5 \mathrm{~mm}, 4.5 \mathrm{~mm}$ and $4,7 \mathrm{~mm}$ 
and these infants were 12,10 and 2 weeks respectively. Their livers were large, harsh and heterogenous in echopattern on ultrasound. The other 5 did not consent to operation though findings on ultrasound suggested biliary atresia. Two of the neonates died, one of whom had a concomitant a gall stone.

\section{Discussion}

It is very important to promptly diagnose surgically correctable disorders, such as biliary atresia, to avoid progressive damage to the liver. However, infantile cholestasis continues to represent a diagnostic challenge in our environment. Magd et al [8] have recently suggested that the TC sign is a simple and useful tool in the diagnosis of biliary atresia. If liver failure or progressive hepatic damage is imminent, early referral to a liver transplant center is advised. Despite the increasing experience and excellent results of pediatric liver transplantation; at this point, surgical corrective procedures such as the Kasai procedure remains the first line of treatment for most patients with biliary atresia [9].

Although the pathogenesis of biliary atresia is still unknown, the disease is characterized by luminal obstruction of the extrahepatic bile duct with a fibrous ductal remnant, which represents the obliterated ductal remnant in the porta hepatis at surgery $[4,7]$. This results in a fibrous tissue at the bifurcation of the portal vein, at the porta hepatis forming a triangular or tubular shaped echogenic density, called "triangular cord" (TC), in the vicinity of the portal vein on a transverse or longitudinal scan [7]. The TC sign, therefore can reliably lead to the exclusion or confirmation of the presence of biliary atresia.

There are 2 distinct clinical types of biliary atresia, fetal-embryonic (appears in the first 2 weeks of life), 10-35\% and perinatal forms (in infants and neonates aged 2-8weeks, 65-90\%) [3] Jaundice was claimed to commence at 10 weeks in most of the patients in this study as reported by their mothers.

Hee-Jung et al [7] and Tan et al [10] also claim that the TC sign and measuring the gallbladder length together are noninvasive, inexpensive, and very useful markers for BA. Park et al [11] opined that using ultrasonography is a new and noninvasive diagnostic tool for the early and definite diagnosis of biliary atresia.

In the present study, only $37.5 \%$ of the TC sign diagnosed by USS were confirmed at surgery. Only one of the study subjects diagnosed as positive had liver cirrhosis which was confirmed by histology. This small percentage might be due to the fact that only 3 of the mothers consented to operation. This could be attributed to their educational status. Being semi literates, they did not deem it a matter of urgency or necessity for the operation and they also reported late to the hospital. The $60 \%$ cases that were TC negative on USS were followed up and managed conservatively, as was the $48 \%$ cases that were ultrasonically diagnosed as hepatitis, by monitoring their clinical and laboratory parameters.

Green and Carroll [12] opined that the demonstration of a decrease in gallbladder size following feeding virtually eliminates the diagnosis of biliary atresia, even in children with abnormal HIDA scans. Only one of the patients in this study did not show a change in the gall bladder size on feeding and she died even before surgery was contemplated. The participants that were proven at surgery to have biliary atresia all did not have their gall bladder demonstrated on USS. This was also confirmed at surgery. Thus supporting the postulation that absence of the gallbladder with a positive TC sign is highly predictive of BA.

The degree of hepatomegaly and heterogeneity was not related to the age but with the degree of liver fibrosis suggesting that the size of the liver and changes in parenchyma echo could indicate the course and prognosis of biliary atresia [5]. This was corroborated in the present study where $72 \%$ of the liver were large and 52\% harsh, though homogenous (88\%) in echopattern. All the proven BA cases had enlarged; harsh echopattern liver and they were aged 2, 10 and 12 weeks. One of them was found to be cirrhotic even by histology.

Ikeda et al [13], performed serial ultrasonic examinations to differentiate biliary atresia from neonatal hepatitis in 144 children, 100 normal neonates and infants, 31 patients with neonatal hepatitis and 13 patients with biliary atresia. They were examined by ultrasound before, during and after feeding. In 97 out of 100 normal children and all patients with neonatal hepatitis, the gallbladder was identified, and the change in size following oral feeding was observed, similar to the findings in this study where all but one of the gallbladder seen on US changed sizes after feeding. However 9 of the gallbladders were not demonstrated in this study. We therefore may postulate that the presence of the gallbladder is also important in diagnosis of hepatitis and its absence may further support the diagnosis of BA, as all the operated cases did not reveal gall bladders as was suggested on USS. Ikeda et al,[13] in four children with severe neonatal hepatitis which could not be differentiated from biliary atresia by clinical and laboratory data, readily identified the gallbladder and observed the change in the size following oral feeding, as was seen in this study. In 8 of 13 of their patients with biliary atresia, they identified a small gallbladder whose size was not affected by oral feeding, as confirmed in one of the patients in the present study. In the other patients the gallbladder was not identified before, during or after oral feeding. On the basis of these results, they consider that serial ultrasonic examination with oral feeding aids in a differential diagnosis of biliary atresia and neonatal hepatitis which we also corroborated in this study. 
Visrutaratna et al [14] claim that the ultrasonographic triangular cord sign coupled with abnormal gallbladder length is more reliable than the ultrasonographic triangular cord sign alone or gallbladder wall irregularity in the diagnosis of biliary atresia as was found in this study where all three subjects that had a positive TC sign on USS, were confirmed at surgery and none of them had gallbladder demonstrated.

When we compare the findings in the present study to that of Shi-Xing lee et al[2] who retrospectively analyzed the sonograms of 20 children with biliary atresia, TC in 10 cases was $30-60 \mathrm{~mm}, 2$ cases did not have a demonstrable gall bladder, all liver showed hepatomegaly and were heterogenous and they concluded that the degree of heterogenicity and hepatomegaly was proportional to liver fibrosis and able to indicate the duration and prognosis of the disease[2,13]. The mean TC width in the present study was $2.62 \pm 1.23 \mathrm{~mm}$ with 9 of our study subjects having TC width between $3.5-4.7 \mathrm{~mm}$. The upper limit of the TC in Shi-Xing lee et al[2] study was much higher when compared to our study. Contrary to Shi-Xing lee et al[2] study, 9 of the cases in this study did not have a demonstrable GB and 2 had small GB. Also, only $72 \%$ had hepatomegaly and only $12 \%$ of the liver was heterogeneous in this study. This may indicate that only these three had significant degree of liver fibrosis and probably cirrhosis. Only one cirrhosis case was however proven by histology in the present study. Several imaging modalities have been used in the diagnosis of BA. Although some findings are highly suggestive of the disease, none is pathognomonic, and reliance on more than one test is common. $[\mathbf{3 , 1 5}]$

Ultrasonography is often the initial investigation in patients with suspected biliary atresia, followed by hepatobiliary scintigraphy, a study that has been used effectively for many years. If the diagnosis remains elusive after these studies, magnetic resonance cholangiopancreatography (MRCP) may be helpful, though they have their limitations $[\mathbf{2 , 3 , 1 5}$. The latter three modes of investigation are expensive and not readily available in our community, thus the relevance and usefulness of ultrasound scan in this environment for diagnosing BA. Endoscopic retrograde cholangiopancreatography is the most useful diagnostic procedure available for direct observation of the extrahepatic bile duct, but it is an invasive procedure that requires the use of general anesthesia, which must be performed by a well-trained endoscopist with the correct instrumentation and technique [15]. When the diameter of the biliary tract is too small, it can be misdiagnosed as biliary atresia.

The advantages of ultrasonography include the fact that the neonatal abdominal wall is very thin, the process is repeatable without any deleterious effect, convenient and non-invasive.[2] However, triangular cord may be absent if (1) there is no TC at the porta hepatis (2) TC is too small to be seen and (3) due to an inexperienced sinologist [2]. This therefore emphasizes the need for adequate training in this field.

We should also not forget that Radionuclide imaging of the liver and gallbladder can sometimes give false positive results [2].

Liver biopsy is often used to confirm the diagnosis of biliary atresia and may be done at the same time as surgical or percutaneous cholangiography [2]. Surgical cholangiography is typically performed by injecting contrast material through the gallbladder. If no communication is seen between the biliary tree and the gastrointestinal tract, biliary atresia is diagnosed [2]. Percutaneous transhepatic cholangiography may be technically challenging, and the results are definitive only if a normal intrahepatic and extrahepatic biliary system is seen.

Ultrasonography-guided percutaneous cholecystocholangiography is a relatively new technique in which radiographic contrast material is injected into the gallbladder under ultrasonographic guidance and the extrahepatic biliary system is viewed with fluoroscopy[3]. Although invasive, the technique has distinct advantages in that it is easier to perform and does not require general anesthesia[3].

In a study by Myung-Joon et al [15], they found that in biliary atresia, T2-weighted single-shot MR cholangiography can show a triangular area of high signal intensity in the porta hepatis that may represent cystic dilatation of the fetal bile duct.

Magnetic resonance (MR) cholangiography can be applied in pediatric patients with neonatal cholestasis or choledochal cysts. It can show a normal extrahepatic bile duct, a dilated common bile duct, and the presence of a choledochal cyst and can be used to exclude biliary atresia as the cause of neonatal cholestasis when the extrahepatic bile duct is observed [15]. This procedure is not readily accessible in our community because it is very expensive and there is limited expertise in this regard.

Johnson et al [1] in a prospective study of 101 Nigerian infants with conjugated hyperbilirubinaemia found that most of the infants presented late, as was seen in the present study, and the superimposed features of prolonged cholestasis made differentiation of the probable causes difficult. In their study, $75 \%$ of patients with sepsis treated with antibiotics, and $70 \%$ of those with hepatitis treated symptomatically, recovered.

Choi et al [4] in their study of 18 jaundiced patients compared the ultrasonographic examination with the histopathologic examination (HPE) of liver specimens obtained from a needle biopsy. The TC was identified in nine patients, all of whom were confirmed to have BA by HPE. In the present study, only one of the 3 confirmed cases had a positive histology report. In Choi et al's[4] study, the TC was not observed in the other nine patients, who had neonatal hepatitis (NH). The mean size of the TC was $13 \mathrm{~mm}$ (wide) x $6 \mathrm{~mm}$ (thick) 
(width range, 5 to $21 \mathrm{~mm}$; thickness range, 4 to $12 \mathrm{~mm}$ ). These findings are at variance with what our study revealed, with a TC mean width of $2.6 \mathrm{~mm}$ and a range of $0.07-4.7 \mathrm{~mm}$.

Nemati et al [16], in a prospective study of 49 infants with prolonged jaundice, between January 2005 April 2007 in Tabriz Children's Hospital using ultrasonography and isotope scan and finally biopsy in all of them under guide of sonography. In their sonographic evaluation, absence or presence of gall bladder, its size and also its evacuation after feeding was checked and beside this triangular cord sign was controlled. The overall accuracy, PPV, NPV, specificity and sensitivity of ultrasonographic findings in diagnosis of EHBA was about 94, 92, 94, 97 and $86 \%$ respectively. They concluded that Ultrasonography is a reliable screening method in early diagnosis of EHBA and triangular cord sign increases its accuracy especially its specificity.

A study done in Thai infants revealed that neonatal hepatitis and extrahepatic biliary atresia were the most common causes of neonatal cholestasis and there were more males than females[17], contrary to the findings in this study where females were more.

Shah et al and Choi et al[4,9] claim that surgical corrective procedures such as the Kasai hepatoportojejunostomy procedure remain the first line of treatment for most patients with EHBA. Although various treatment options are being tried for biliary atresia, the primary treatment is surgery, by resection of the fibrous bile duct remnant, followed by a Roux-en-Y anastomosis of the bowel to the bed of the porta hepatis, according to the Kasai portoenterostomy procedure [3], which was done in all three patients in this study.

Kotb et al[18] studied 27 infants Post-portoenterostomy and concluded that TC sign is associated with more morbidity and mortality; and reflects inadequate surgical technique. Therefore post operative monitoring of the TC sign might give an indication of the success of the operation apart from the clinical assessment.

\section{Conclusion}

In conclusion, we propose that the TC sign be defined as a triangular or tubular echogenic structure anterior to the bifurcation of the portal vein, and is highly suggestive of biliary atresia when it is $3.5-4 \mathrm{~mm}$ and above on a transverse or longitudinal scan especially when the gall bladder is absent.

This study has also revealed that the identification of the gall bladder is an integral part of excluding biliary atresia on ultrasound scan aside from demonstrating the fibrous ductal remnant in the porta hepatis, TC sign. A small GB might however suggest incomplete obstruction of the biliary tree.

The TC sign is a non invasive, rapid, simple, and reliable diagnostic means for the assessment of infants with persistent conjugated hyperbilirubinaemia. We recommend this diagnostic test for patients suspected to have biliary atresia, suggesting that all such patients should have an abdominal scan.

\section{Limitations}

1. The educational status of the parents and their level of awareness about BA made it very difficult for us to be able to convince them to agree to operate on the infants.

2. The high cost of medical care and administrative bureaucracy in the hospital contributed to the loss of some of the cases to follow up.

\section{Recommendations}

1. Mothers of babies discharged post-delivery should be enlightened about persistent jaundice to increase their level of awareness about BA and be encouraged to return to the paediatric clinic once they notice jaundice to reduce morbidity and mortality from BA.

2. All neonates with persistent jaundice should have an abdominal scan to screen for the TC sign to rule out biliary atresia.

\section{References}

[1] AO Johnson, VA Nottidge, CO Ojo, TA Junaid, NA Akingbehin, EB Attah_Conjugated hyperbilirubinaemia in Nigerian infants, Afr J Med Med Sci. 9(3-4), 1980, 117-127.

[2] Shi-Xing Li, Yao Zhang, Mei Sun, Bo Shi, Zhong-Yi Xu, Ying Huang, and Zhi-Qin Mao Ultrasonic diagnosis of biliary atresia: a retrospective analysis of 20 Patients. World J Gastroenterol.14 (22),2008, 3579-3582.

[3] K . Zukotynski, EC Lin. Biliary Atresia. Medscape reference, retreived from http://emedicine.medscape.com/article/406335overview.

[4] SO Choi , WH Park, HJ Lee, SK Woo. 'Triangular cord': a sonographic finding applicable in the diagnosis of biliary atresia. J Pediatr Surg. 31(3), 1996, 363-366.

[5] Y Weerakkody and G Singh et al. Biliary atresia. Retrieved from Radiopedia.org http://radiopaedia.org/articles/biliary-atresia.

[6] MH Imanieh, SM Dehghani, MH Bagheri, V Emad, M Haghighat, M M Zahmatkeshan, HR Forutan, AR Rasekhi, F Gheisari .Triangular cord sign in detection of biliary atresia: is it a valuable sign? Dig Dis Sci. 55(1),2010,172-175. doi: $10.1259 / \mathrm{bjr} / 34728497$.

[7] Hee-Jung Lee, Sung-Moon Lee, Woo-Hyun Park, Soon-Ok Choi. Objective Criteria of Triangular Cord Sign in Biliary Atresia on US Scans. doi: 10.1148/radiol.292020472 Radiology. 229. 2003, 395-400. 
[8] A. K Magd, K Ahmed, F. S Maha , M. E Nehal , M. E Hanaa, K. A Mohamed , A Ahmed, EE Mohammed, W Rady , M Hosam , K Manoochehr , H. A Hassan . Evaluation of the Triangular Cord Sign in the Diagnosis of Biliary Atresia Pediatrics.108 (2), 2001 , $416-420$.

[9] HA Shah, W Spivak . Neonatal cholestasis. New approaches to diagnostic evaluation and therapy. Pediatr Clin North Am. 41(5), 1994, 943-966.

[10] AP Tan Kendrick , KB Phua , BC Ooi , R Subramaniam , CE Tan, AS Goh . Making the diagnosis of biliary atresia using the triangular cord sign and gallbladder length. Pediatr Radiol. 30(2), 2000, 69-73.

[11] WH Park, SO Choi , HJ Lee, SP Kim, SK Zeon, SL Lee. A new diagnostic approach to biliary atresia with emphasis on the ultrasonographic triangular cord sign: comparison of ultrasonography, hepatobiliary scintigraphy, and liver needle biopsy in the evaluation of infantile cholestasis. J Pediatr Surg. 32(11), 1997, 1555-1559.

[12] D Green, BA Carroll. Ultrasonography in jaundiced infant: a new approach. JUM. 5(6), 1986, 323-329.

[13] S Ikeda, Y Sera, M Akagi. Serial ultrasonic examination to differentiate biliary atresia from neonatal hepatitis--special reference to changes in size of the gallbladder. Eur J Pediatr.148(5), 1989, 396-400.

[14] P Visrutaratna, L Wongsawasdi, P Lerttumnongtum, J Singhavejsakul, V Kattipattanapong, N $\underline{\text { Ukarapol }}$. Triangular cord sign and ultrasound features of the gall bladder in infants with biliary atresia. Australas Radiol. 47(3), 2003, 252-6.

[15] K Myung-Joon, P Young Nyun, H Seok Joo, SY Choon, S Y Hyung, HH Eui, SC Ki . Biliary Atresia in Neonates and Infants: Triangular Area of High Signal Intensity in the Porta Hepatis at T2-weighted MR Cholangiography with US and Histopathologic Correlation. Radiology. 215, 2000, 395-401.

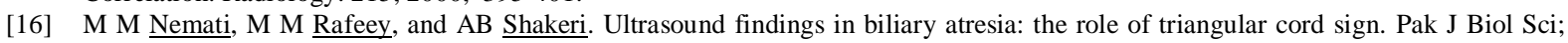
12(1), 2009, 95-97

[17] P Aanpreung, M Laohapansang, R Ruangtrakool, J Kimhan. Neonatal cholestasis in Thai infants. J Med Assoc Thailand. 88, 2005, S8, S9-S15.

[18] M A Kotb, , M Sheba, N Koofy S El, Mansour, H M El Karaksy, N M Dessouki, W Mostafa, M El Barbary, H E El-Tantawy, S Kaddah. Post-portoenterostomy triangular cord sign prognostic value in biliary atresia: a prospective study. British Journal of Radiology 78, 2005, 884-887. doi: 10.1542/peds.108.2.416. 